Research Article

\title{
On the Spectrum of Laplacian Matrix
}

\author{
Akbar Jahanbani $(\mathbb{D}$, Seyed Mahmoud Sheikholeslami $(\mathbb{D}$, and Rana Khoeilar
}

Department of Mathematics, Azarbaijan Shahid Madani University, Tabriz, Iran

Correspondence should be addressed to Akbar Jahanbani; akbar.jahanbani92@gmail.com

Received 12 April 2021; Accepted 19 May 2021; Published 4 June 2021

Academic Editor: Andrea Semaničová-Feňovčíková

Copyright ( $\odot 2021$ Akbar Jahanbani et al. This is an open access article distributed under the Creative Commons Attribution License, which permits unrestricted use, distribution, and reproduction in any medium, provided the original work is properly cited.

Let $G$ be a simple graph of order $n$. The matrix $\mathscr{L}(G)=D(G)-A(G)$ is called the Laplacian matrix of $G$, where $D(G)$ and $A(G)$ denote the diagonal matrix of vertex degrees and the adjacency matrix of $G$, respectively. Let $l_{1}(G), l_{n-1}(G)$ be the largest eigenvalue, the second smallest eigenvalue of $\mathscr{L}(G)$ respectively, and $\lambda_{1}(G)$ be the largest eigenvalue of $A(G)$. In this paper, we will present sharp upper and lower bounds for $l_{1}(G)$ and $l_{n-1}(G)$. Moreover, we investigate the relation between $l_{1}(G)$ and $\lambda_{1}(G)$.

\section{Introduction}

We begin with the preliminaries which are required throughout this paper. Let $G$ be a simple graph with vertex set $V=V(G)$ and edge set $E(G)$. The integers $n=n(G)=|V(G)|$ and $\varepsilon=$ $\mathcal{E}(G)=|E(G)|$ are the order and the size of the graph $G$, respectively. The open neighborhood of vertex $v_{i}$ is $N_{G}\left(v_{i}\right)=N\left(v_{i}\right)=\left\{v_{j} \in V(G) \mid v_{i} v_{j} \in E(G)\right\}$, and the degree of $v_{i}$ is $d_{G}\left(v_{i}\right)=d_{i}=\left|N\left(v_{i}\right)\right|$. Let $K_{n}$ be the complete graph of order $n$ and $\bar{G}$ be the complement of the graph $G$. Let $\Delta$ and $\delta$ be the maximum degree and the minimum degree of the vertices of $G$, respectively. The eigenvalues of the adjacency matrix $A(G)$, are denoted by $\lambda_{1}(G) \geq \lambda_{2}(G) \geq \ldots \geq \lambda_{n}(G)$. The matrix $\mathscr{L}(G)=D(G)-A(G)$, where $D(G)$ is the diagonal matrix of vertex degrees, is called the Laplacian matrix of $G$ and rarely appears in the literature. The eigenvalues of Laplacian matrix $G$ are denoted as $l_{1}(G) \geq l_{2}(G) \geq \ldots \geq l_{n}(G)=0$. The Laplacian matrix of a graph and its eigenvalues can be used in several areas of mathematical research and have a physical interpretation in various physical and chemical theories. The adjacency matrix of a graph and its eigenvalues were much more investigated in the past than the Laplacian matrix. Many related physical quantities have the same relation to $\mathscr{L}(G)$; also, there are many problems in physics and chemistry where the Laplacian matrices of graphs and their spectra play the central role. Recently, its applications to several difficult problems in graph theory were discovered (see [1-7]).
Merris [8] discussed the Laplacian matrices of graphs. In [9], some bounds are established for Laplacian eigenvalues of graphs. Taheri et al. [10] presented some bounds for the largest Laplacian eigenvalue of graphs. Patra et al. [11] obtained bounds for the Laplacian spectral radius of graphs. In [12], the authors investigated some bounds for the Laplacian spectral radius of an oriented hypergraph. Chen [13] established some bounds for $\lambda_{1}(G)$.

In this paper, we first present sharp upper and lower bounds for $l_{1}(G)$ and $l_{n-1}(G)$, and then we investigate the relation between $l_{1}(G)$ and $\lambda_{1}(G)$.

\section{Preliminaries}

In this section, some fundamental results that are used in this paper are recalled. We begin with the following result, which plays a key role in this section.

Lemma 1 (see [14]). Let $G$ be a graph of order $n$ and size $\varepsilon$. Then,

$$
\begin{aligned}
& \sum_{i=1}^{n-1} l_{i}=\sum_{i=1}^{n} l_{i}=\operatorname{tr} \mathscr{L}(G)=2 \varepsilon, \\
& \sum_{i=1}^{n-1} l_{i}^{2}=\sum_{i=1}^{n} l_{i}^{2}=\operatorname{tr} \mathscr{L}^{2}(G)=2 \varepsilon+M_{1}(G),
\end{aligned}
$$


where $M_{1}(G)=\sum_{i=1}^{n} d_{i}^{2}$ is the well-known graph invariant called the first Zagreb index [15].

Favaron and Mahéo [16] proved the following result:

Lemma 2 (see [16]). Let $G$ be a graph of order $n$. Then,

$$
\lambda_{1} \geq \sqrt{\frac{\sum_{i=1}^{n} d_{i}^{2}}{n}}
$$

The proof of the next result can be found in [17].

Lemma 3. Let $G$ be a graph of order $n$. Then, $l_{1}=l_{2}=\ldots=$ $l_{n-1}$ if and only if $G \cong K_{n}$ or $G \cong \bar{K}_{n}$.

Das in [18] proved the following lemma.

Lemma 4. Let $G$ be a connected graph of order $n \geq 3$. Then, $l_{2}=l_{3}=\ldots=l_{n-1}$ if and only if $G \cong K_{n}$.

In [14], a class of real polynomials $P_{n}(x)=x^{n}+a_{1} x^{n-1}+$ $a_{2} x^{n-2}+b_{3} x^{n-3}+\ldots+b_{n}$, denoted as $\mathscr{P}_{n}\left(a_{1}, a_{2}\right)$, where $a_{1}$ and $\mathrm{a}_{2}$ are fixed real numbers, was considered.

Theorem 1. For the roots $y_{1} \geqslant y_{2} \geqslant \ldots \geqslant y_{n}$ of an arbitrary polynomial $\varphi_{n}(y)$ from this class, the following values were introduced:

$$
\begin{aligned}
& \bar{y}=\frac{1}{n} \sum_{i=1}^{n} y_{i}, \\
& \Gamma=n \sum_{i=1}^{n} y_{i}^{2}-\left(\sum_{i=1}^{n} y_{i}\right)^{2} .
\end{aligned}
$$

Then upper and lower bounds for the polynomial roots, $y_{i}, i=1,2, \ldots, n$, were determined in terms of the introduced values

$$
\begin{aligned}
& \bar{y}+\frac{1}{n} \sqrt{\frac{\Gamma}{n-1}} \leqslant y_{1} \leqslant \bar{y}+\frac{1}{n} \sqrt{(n-1) \Gamma}, \\
& \bar{y}-\frac{1}{n} \sqrt{\frac{(i-1)}{n-i+1}} \Gamma \leqslant y_{i} \leqslant \bar{y}+\frac{1}{n} \sqrt{\frac{(n-i)}{i}} \Gamma, \quad \text { for } i=2,3, \ldots, n-1 .
\end{aligned}
$$

\section{Main Results}

In this section, we will obtain some sharp upper and lower bounds for $l_{1}(G)$ and $l_{n-1}(G)$ involving the first Zagreb index and order and size of graphs. Moreover, we investigate the relation between $l_{1}(G)$ and $\lambda_{1}(G)$. The first result is an immediate consequence of Theorem 1 and Lemma 1.

Lemma 5. Let $G$ be a graph of order $n \geq 2$ and size $\varepsilon$. Then,

$$
\begin{gathered}
\frac{2 \varepsilon+\sqrt{(n-1)\left(2 \varepsilon+M_{1}(G)\right)-4 \varepsilon^{2} / n-2}}{n-1} \leq l_{1} \\
\leq \frac{2 \varepsilon+\sqrt{(n-2)\left((n-1)\left(2 \varepsilon+M_{1}(G)\right)-4 \varepsilon^{2}\right)}}{n-1}, \\
\frac{2 \varepsilon-\sqrt{(n-2)\left((n-1)\left(2 \varepsilon+M_{1}(G)\right)-4 \varepsilon^{2}\right) / 2}}{n-1} \leq l_{n-1} \leq \frac{2 \varepsilon}{n-1} .
\end{gathered}
$$

Here, we will obtain a lower and an upper bound for the largest Laplacian eigenvalue $l_{1}$ and the second smallest Laplacian eigenvalue $l_{n-1}$, respectively.

Theorem 2. Let $G$ be a graph of order $n \geq 3$ and size $\varepsilon$. Then,

$$
l_{1} \geq \frac{2 \varepsilon}{n-1}+\sqrt{\frac{1}{(n-1)(n-2)}\left(\frac{2 \varepsilon(n-1+2 \varepsilon)}{n-1}+\sum_{i=1}^{n} d_{i}^{2}\right)},
$$

$$
l_{n-1} \leq \frac{2 \varepsilon}{n-1}-\sqrt{\frac{1}{(n-1)(n-2)}\left(\frac{2 \varepsilon(n-1+2 \varepsilon)}{n-1}+\sum_{i=1}^{n} d_{i}^{2}\right)},
$$

and the equalities hold if and only if $G \cong K_{n}$ or $G \cong \bar{K}_{n}$.

Proof. For every fixed number $t$, we can write that

$$
\begin{aligned}
\left(\sum_{i=1}^{n-1} l_{i}-(n-1) l_{t}\right)^{2} & =\left(\sum_{i=1}^{n-1}\left(l_{i}-l_{t}\right)\right)^{2} \\
& =\sum_{i=1}^{n-1}\left(l_{i}-l_{t}\right)^{2}+2 \sum_{1 \leq i \leq j \leq n-1}\left(l_{i}-l_{t}\right)\left(l_{i}-l_{t}\right) .
\end{aligned}
$$

It is not hard to see that when $t=1$ or $t=n-1$, we get

$$
\sum_{1 \leq i \leq j \leq n-1}\left(l_{i}-l_{t}\right)\left(l_{i}-l_{t}\right) \geq 0 .
$$

Hence, we have

$$
\left(\sum_{i=1}^{n-1} l_{i}-(n-1) l_{t}\right)^{2} \geq \sum_{i=1}^{n-1}\left(l_{i}-l_{t}\right)^{2} .
$$

So, we can write 


$$
\begin{aligned}
& \left(\sum_{i=1}^{n-1} l_{i}\right)^{2}-2(n-1) l_{t} \sum_{i=1}^{n-1} l_{i}+(n-1)^{2} l_{t}^{2} \\
& \geq \sum_{i=1}^{n-1} l_{i}^{2}-2 l_{t} \sum_{i=1}^{n-1} l_{i}+(n-1) l_{t} .
\end{aligned}
$$

This is equivalent to

$$
l_{t}^{2}-\frac{2 l_{t} \sum_{i=1}^{n-1} l_{i}}{n-1}+\frac{\left(\sum_{i=1}^{n-1} l_{i}\right)^{2}}{(n-1)^{2}-(n-1)} \geq \frac{\sum_{i=1}^{n-1} l_{i}^{2}}{(n-1)^{2}-(n-1)},
$$

or

$$
\left(l_{t}-\frac{\sum_{i=1}^{n-1} l_{i}}{n-1}\right)^{2}-\frac{\left(\sum_{i=1}^{n-1} l_{i}\right)^{2}}{(n-1)^{2}}+\frac{\left(\sum_{i=1}^{n-1} l_{i}\right)^{2}}{(n-1)(n-2)} \geq \frac{\sum_{i=1}^{n-1} l_{i}^{2}}{(n-1)(n-2)} .
$$

Therefore, we have

$$
\left(l_{t}-\frac{\sum_{i=1}^{n-1} l_{i}}{n-1}\right)^{2} \geq \frac{1}{(n-1)(n-2)}\left(\sum_{i=1}^{n-1} l_{i}^{2}-\frac{1}{n-1}\left(\sum_{i=1}^{n-1} l_{i}\right)^{2}\right) \text {. }
$$

Hence, by using Lemma 1, we have

$$
\begin{aligned}
& \sum_{i=1}^{n-1} l_{i}=\sum_{i=1}^{n} l_{i}=\operatorname{tr} \mathscr{L}(G)=2 \varepsilon, \\
& \sum_{i=1}^{n-1} l_{i}^{2}=\sum_{i=1}^{n} l_{i}^{2}=\operatorname{tr} \mathscr{L}^{2}(G)=2 \varepsilon+M_{1}(G) .
\end{aligned}
$$

By combining inequalities (15)-(17), we get the following inequality:

$$
\left(l_{1}-\frac{2 \varepsilon}{n-1}\right)^{2} \geq \frac{1}{(n-1)(n-2)}\left(2 \varepsilon-\frac{4 \varepsilon^{2}}{n-1}+\sum_{i=1}^{n} d_{i}^{2}\right) .
$$

By inequalities (5) and (6), we have

$$
l_{1}-\frac{2 \varepsilon}{n-1} \geq 0, l_{n-1}-\frac{2 \varepsilon}{n-1} \leq 0 .
$$

Therefore, we have

$$
\begin{gathered}
l_{1} \geq \frac{2 \varepsilon}{n-1}+\sqrt{\frac{1}{(n-1)(n-2)}\left(\frac{2 \varepsilon(n-1+2 \varepsilon)}{n-1}+\sum_{i=1}^{n} d_{i}^{2}\right)} \\
l_{n-1} \leq \frac{2 \varepsilon}{n-1}-\sqrt{\frac{1}{(n-1)(n-2)}\left(\frac{2 \varepsilon(n-1+2 \varepsilon)}{n-1}+\sum_{i=1}^{n} d_{i}^{2}\right)} .
\end{gathered}
$$

If the equality in (7) holds, then the inequality in (10) must hold, and hence we have $l_{1}=l_{2}=\cdots=l_{n-1}=2 \varepsilon / n-1$; thus, by Lemma 3 , we have $G \cong K_{n}$ or $G \cong \bar{K}_{n}$. Conversely, if
$G \cong K_{n}$ or $G \cong \bar{K}_{n}$, then it is not difficult to see that the equalities in (7) and (8) hold.

Next, we present an upper bound for spectral radius of the Laplacian matrix.

Theorem 3. Let $G$ be a connected graph of order $n \geq 2$ and size $\varepsilon$. Then,

$$
l_{1} \leq \frac{\sqrt{(16 n-16)\left(\left(2 \varepsilon+M_{1}(G)\right)(n-2)-4 \varepsilon^{2}\right)}+8 \varepsilon}{8 n-8} .
$$

Proof. Applying Lemma 1, we can write

$$
\beta:=\sum_{i=1}^{n-1} l_{i}^{2}=2 \varepsilon+\sum_{i=1}^{n-1} d_{i}^{2}=2 \varepsilon+M_{1}(G),
$$

or

$$
l_{1}^{2}=\beta-\sum_{i=2}^{n-1} l_{i}^{2} \leq \beta-\frac{1}{n-2}\left(\sum_{i=2}^{n-1} l_{i}\right)^{2}=\beta-\frac{\left(2 \varepsilon-l_{1}\right)^{2}}{n-2} .
$$

By inequality (23), we have

$$
l_{1}^{2} \leq \beta-\frac{4 \varepsilon^{2}+l_{1}^{2}-4 \varepsilon l_{1}}{n-2} .
$$

Using inequality (24), we get

$$
l_{1}^{2}\left(1+\frac{1}{n-2}\right)+\frac{4 \varepsilon^{2}}{n-2}-\frac{4 \varepsilon l_{1}}{n-2}-\beta \leq 0,
$$

or

$$
l_{1}^{2}(n-1)+4 \varepsilon^{2}-4 \varepsilon l_{1}-(n-2) \beta \leq 0 .
$$

By inequality (26), we can write

$$
l_{1}^{2}(n-1)+4 \varepsilon^{2}-4 \varepsilon l_{1}-2 \varepsilon(n-2)-M_{1}(G)(n-2) \leq 0 .
$$

Solving this inequality leads to

$$
l_{1} \leq \frac{\sqrt{(16 n-16)\left(\left(2 \varepsilon+M_{1}(G)\right)(n-2)-4 \varepsilon^{2}\right)}+8 \varepsilon}{8 n-8} .
$$

Finally, we will describe a relationship between spectral radius $\left(l_{1}\right)$ of the Laplacian matrix and the spectral radius $\left(\lambda_{1}\right)$ of the adjacency matrix.

Theorem 4. Let $G$ be a connected graph of order $n \geq 3$ and size $\varepsilon$. Then,

$$
\lambda_{1} \geq \sqrt{\frac{l_{1}^{2}(n-1)}{n(n-2)}+\frac{4 \varepsilon^{2}}{n(n-2)}-\frac{4 \varepsilon l_{1}}{n(n-2)}-\frac{2 \varepsilon}{n}},
$$

and the equality holds if and only if $G \cong K_{n}$.

Proof. By inequality (26) and Lemma 2, we have 


$$
\begin{gathered}
l_{1}^{2}(n-1)+4 \varepsilon^{2}-4 \varepsilon l_{1} \leq(n-2) \beta=(n-2)\left(2 \varepsilon+\sum_{i=1}^{n} d_{i}^{2}\right), \\
\leq(n-2)\left(2 \varepsilon+n \lambda_{1}^{2}\right)=2 \varepsilon(n-2)+n(n-2) \lambda_{1}^{2} .
\end{gathered}
$$

Now suppose that the equality holds in (29). Then, all the inequalities in the proof must be equalities.

If the equality holds in (30), then inequality (23) must be equality; in other words,

$$
\beta-\sum_{i=2}^{n-1} l_{i}^{2}=\beta-\frac{1}{n-2}\left(\sum_{i=2}^{n-1} l_{i}\right)^{2},
$$

or

$$
\sum_{i=2}^{n-1} l_{i}^{2}=\frac{1}{n-2}\left(\sum_{i=2}^{n-1} l_{i}\right)^{2}
$$

Therefore, by equality (33), we get

$$
l_{2}=l_{3}=\cdots=l_{n-1} \text {. }
$$

Hence, by Lemma 4 , we get $G \cong K_{n}$. Conversely, one can easily see that equality holds in (29) when $G \cong K_{n}$.

\section{Conclusion}

In this paper, we established some sharp upper and lower bounds for the largest eigenvalue and the second smallest eigenvalues of Laplacian matrix involving the first Zagreb index and order and size of graphs. Moreover, we investigate a relation between the largest eigenvalues of Laplacian matrix and the adjacency matrix.

There are still open and challenging problems for researchers. For example, the problem of ABC matrix, GA matrix, and so on remains open for further investigation.

\section{Data Availability}

The data involved in the examples of our manuscript are included within the article.

\section{Conflicts of Interest}

The authors declare that they have no conflicts of interest.

\section{References}

[1] B. E. Eichinger, "An approach to distribution functions for Gaussian molecules," Macromolecules, vol. 10, no. 3, pp. 671-675, 1977.

[2] B. E. Eichinger, "Scattering functions for Gaussian molecules," Macromolecules, vol. 11, no. no. 2, pp. 432-433, 1978.

[3] B. E. Eichinger, "Scattering functions for Gaussian molecules. 2. Intermolecular correlations," Macromolecules, vol. 11, no. 5, pp. 1056-1057, 1978.

[4] H. A. Ganie, S. Pirzada, and T. Vilmar, "On the sum of $\mathrm{k}$ largest laplacian eigenvalues of a graph and clique number,"
Mediterranean Journal of Mathematics, vol. 18, no. 1, pp. 1-13, 2021.

[5] J. Jost, R. Mulas, and F. Munch, "Spectral gap of the largest eigenvalue of the normalized graph Laplacian," Communications in Mathematics and Statistics, vol. 9, pp. 1-11, 2021.

[6] R. Li, "Combinations of some spectral invariants and Hamiltonian properties of graphs," Contributions to Mathematics, vol. 1, pp. 54-56, 2020.

[7] C. Maas, "Transportation in graphs and the admittance spectrum," Discrete Applied Mathematics, vol. 16, no. 1, pp. 31-49, 1987.

[8] R. Merris, "Laplacian matrices of graphs: a survey," Linear Algebra and Its Applications, vol. 197-198, pp. 143-176, 1994.

[9] S. Buyukkose and S. Basdas, "Bounds for the laplacian eigenvalue of graphs using 2-adjacency," Journal of Science and Arts, vol. 18, no. 1, pp. 175-182, 2018.

[10] H. Taheri and G. H. Fath-Tabar, "New upper bound on the largest laplacian eigenvalue of graphs," Facta Universitatis, Series: Mathematics and Informatics, vol. 35, pp. 533-540, 2020.

[11] K. L. Patra, B. K. Sahoo, and B. K. Sahoo, "Bounds for the Laplacian spectral radius of graphs," Electronic Journal of Graph Theory and Applications, vol. 5, no. 2, pp. 276-303, 2017.

[12] O. Kitouni and R. Nathan, "Lower bounds for the Laplacian spectral radius of an oriented hypergraph," Australasian Journal of Combinatorics, vol. 74, no. 3, pp. 408-422, 2019.

[13] C. Yan, "Properties of spectra of graphs and line graphs," Applied Mathematics-A Journal of Chinese Universities, vol. 17, no. 3, pp. 371-376, 2002.

[14] A. Lupaş, "Inequalities for the roots of a class of polynomials," Publikacije Elektrotehničkog Fakulteta. Serija Matematika I Fizika, vol. 577/598, pp. 79-85, 1977.

[15] I. Gutman and Ch Das Kinkar, "The first Zagreb index 30 years after." MATCH Commun," Math. Comput. Chem, vol. 50, no. no. 1, pp. 83-92, 2004.

[16] O. Favaron and M. Mahéo, "Some eigenvalue properties in graphs (conjectures of Graffiti-II)," Discrete Mathematics, vol. 111, no. 1-3, pp. 197-220, 1993.

[17] Bo Zhou, "On sum of powers of the Laplacian eigenvalues of graphs," Linear Algebra and Its Applications, vol. 429, no. 8-9, pp. 2239-2246, 2008.

[18] K. C. Das, "A sharp upper bound for the number of spanning trees of a graph," Graphs and Combinatorics, vol. 23, no. 6, pp. 625-632, 2007. 